\title{
Genomic scale analysis of lateral gene transfer in Apicomplexan parasites: insights into early eukaryotic evolution, host-pathogen interaction and drug target development Lucia Peixoto* and David S Roos
}

\author{
Address: Department of Biology, University of Pennsylvania, Philadelphia, PA, USA \\ Email: Lucia Peixoto* - luciap@sas.upenn.edu \\ * Corresponding author
}

\begin{abstract}
from Third International Society for Computational Biology (ISCB) Student Council Symposium at the Fifteenth Annual International Conference on Intelligent Systems for Molecular Biology (ISMB)

Vienna, Austria. 21 July 2007
\end{abstract}

Published: 20 November 2007

BMC Bioinformatics 2007, 8(Suppl 8):S5 doi:10.1 186/147|-2105-8-S8-S5

This abstract is available from: http://www.biomedcentral.com//47I-2/05/8/S8/S5

(c) 2007 Peixoto and Roos; licensee BioMed Central Ltd.

The protozoan phylum Apicomplexa is comprised of $>5000$ species, including Toxoplasma gondii (a prominent opportunistic infection in AIDS), and the Plasmodium parasites responsible for malaria. Complete genome sequences are now available for several apicomplexan species, including both $T$. gondii and Plasmodium falciparum, making it possible to exploit comparative phylogenomic tools for insight into adaptations associated with intracellular parasitism, and in order to identify targets for therapeutic development. These parasites harbor a novel organelle, the 'apicoplast', which was acquired by endosymbiotic capture of a eukaryotic alga, and thus provides a dramatic example of lateral gene transfer (LGT). In order to determine the overall impact of LGT, we have employed phylogeny reconstruction to detect transfers on a genomicscale. Trees have been constructed for all genes in the Toxoplasma and Plasmodium genomes, and compared with a whole genome species treederived from 55 taxa represented in the orthologous protein database OrthoMCL-DB [1]. Non-apicomplexan specific ortholog groups containing 4 to 100 sequences were examined, pruning outlying species based on their degree of connectivity to the group as a whole, and robustness of the resulting tree.
A pilot study based on intensive manual curation of $T$. gondii genes coding for metabolic enzymes estimates that $\sim 15 \%$ of these genes were acquired by LGT. Turning to the entire parasite genome(s), Horizstory software identifies 1552 groups where T. gondii gene placement contrasts with the species tree, and 1520 instances of potential LGTinvolving $P$. falciparum. In order to identify the most probable LGT events, we developed a strategy that ranks the probability ofLGT based on the taxonomic consistency of results for sibling species within the tree, and bootstrap support for the relevant internalnode(s). This 'LGTsmart' strategy yields a ranked list of probable lateral gene transfer events (Figure 1) including 226 high confidence events that occurred at different times during the course of apicomplexan evolution. Many of these transfers involve genes exhibiting phylogenetic affinity with plants, consistent with the algal origin of the apicoplast endosymbiont. Additional, more recent, transfers appear to have a bacterial origin, and both classes of genes include promising targets for drug development. Interestingly, we also detected LGT of metazoan-like genes, possibly acquired through interaction with the host during adaptation to the parasitic lifestyle. 


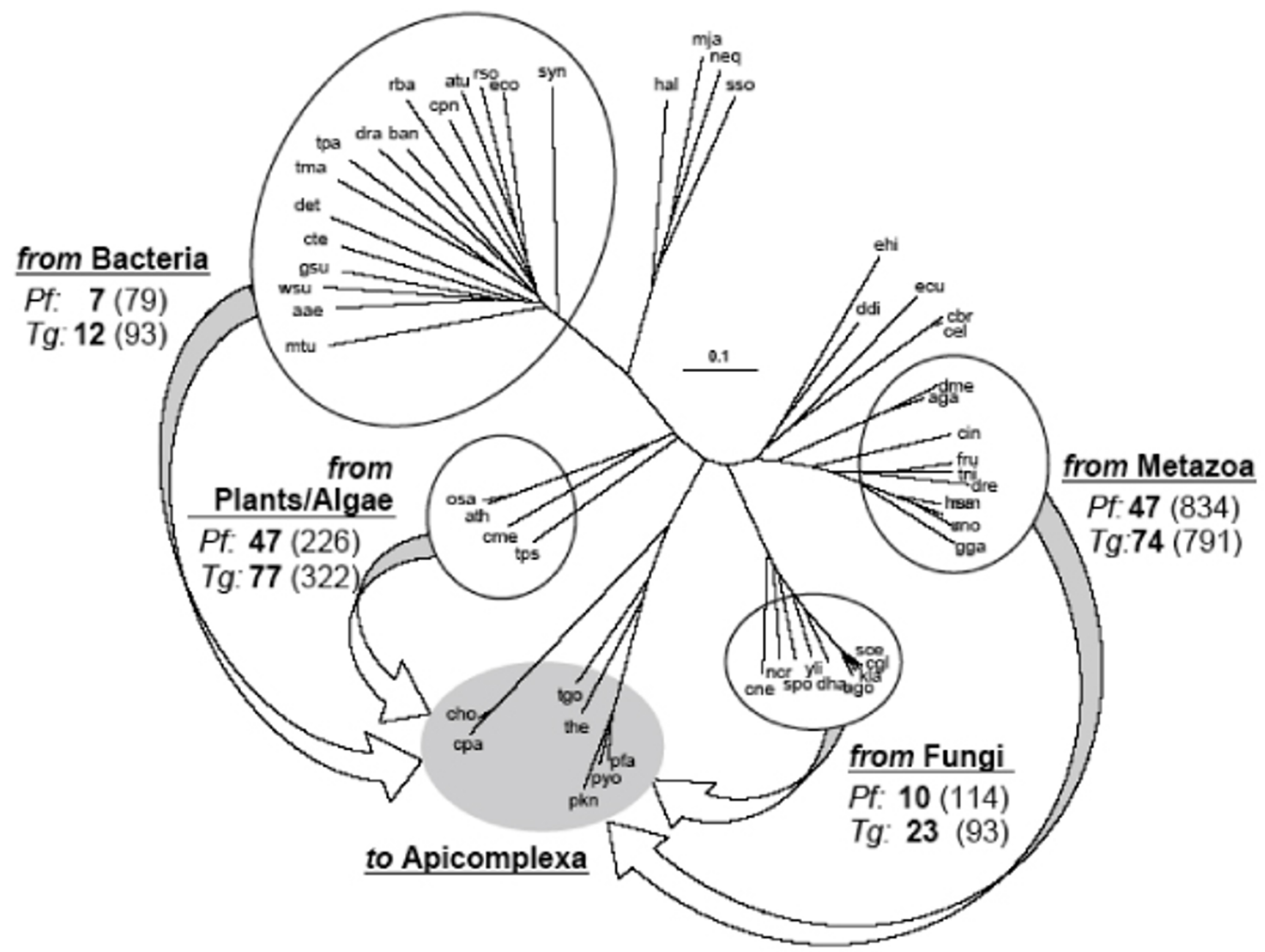

Figure I

Probable source of transfer events identified by the LGT smart algorithm. Parentheses indicate possible LGT events for each source clade; bold indicates high confidence transfers.

\section{References}

I. Chen F, Mackey AJ, Stoeckert C, Roos DS: OrthoMCL-DB: Querying a comprehensive multi-species collection of ortholog groups. Nucleic Acids Res 2006, 34:D363-368.

Publish with Bio Med Central and every scientist can read your work free of charge

"BioMed Central will be the most significant development for disseminating the results of biomedical research in our lifetime."

Sir Paul Nurse, Cancer Research UK

Your research papers will be:

- available free of charge to the entire biomedical community

- peer reviewed and published immediately upon acceptance

- cited in PubMed and archived on PubMed Central

- yours - you keep the copyright
BioMedcentral 